\title{
Correction to: Dual Hashing-Based Algorithms for Discrete Integration
}

\author{
Alexis de Colnet and Kuldeep S. Meel
}

Correction to:

Chapter "Dual Hashing-Based Algorithms for Discrete

Integration" in: T. Schiex and S. de Givry (Eds.):

Principles and Practice of Constraint Programming, LNCS 11802, https://doi.org/10.1007/978-3-030-30048-7_10

In the version of this paper that was originally published, reference was made to an incorrect grant number. This has now been corrected. 